\title{
An unusual presentation of Sweet syndrome in a patient with ulcerative colitis treated with a tumor necrosis factor alpha inhibitor: a case report
}

\author{
Yung-Chun Chang ${ }^{1}$ and Hui-Ju Yang ${ }^{2}$ \\ ${ }^{1}$ Changhua Christian Hospital \\ ${ }^{2}$ Chung Shan Medical University
}

June 12, 2021

\begin{abstract}
Tumor necrosis factor (TNF) alpha inhibitors are widely used to treat inflammatory bowel disease (IBD) and Sweet syndrome is known as an extra-intestinal cutaneous manifestation of IBD. We wish to highlight a paradoxical case and successful management of anti-TNF-agent-induced Sweet syndrome compared with Sweet syndrome treated by anti-TNF agents.
\end{abstract}

(a) Title Page

An unusual presentation of Sweet syndrome in a patient with ulcerative colitis treated with a tumor necrosis factor alpha inhibitor: a case report

Author: Yung-Chun Chang ${ }^{1,2}$, Hui-Ju Yang ${ }^{3,4}$

${ }^{1}$ Institute of Medicine, China Medical University, Taichung, Taiwan

2 Changhua Christian Hospital, Changhua, Taiwan

3 Institute of Medicine, Chung Shan Medical University, Taichung, Taiwan

4 Department of Dermatology, Changhua Christian Hospital, Changhua, Taiwan

Running Head: Unusual presentation of Sweet syndrome

1. [Corresponding Author]: Hui-Ju Yang

2. [Institution]: Department of Dermatology, Changhua Christian Hospital, Changhua, Taiwan

3. [Institution Address]: No. 135, Nanxiao St., Changhua City, Changhua County 500054, Taiwan (R.O.C.)

4. [Author e-mail address]:u103001425@cmu.edu.tw

5. [Corresponding Author E-mail address]:149777@cch.org.tw

6. [Tel]: area code (Taiwan/886) -4-7238595 (extend number 4361)

7. [Fax]: area code (Taiwan/886) -4-7232942

(b) Key clinical message

When distinguishing between extra-intestinal cutaneous manifestation flare-ups and drug-induced skin lesions, anti-TNF agents should be withheld, and steroid doses increased in cases of suspected anti-TNF-agentinduced Sweet syndrome.

(c) Abstract 
Tumor necrosis factor (TNF) alpha inhibitors are widely used to treat inflammatory bowel disease (IBD) and Sweet syndrome is known as an extra-intestinal cutaneous manifestation of IBD. We wish to highlight a paradoxical case and successful management of anti-TNF-agent-induced Sweet syndrome compared with Sweet syndrome treated by anti-TNF agents.

Keyword: Sweet syndrome, ulcerative colitis, tumor necrosis factor alpha inhibitor, adverse event, cytokine dysregulation

(d) Main text

Introduction

Sweet syndrome, also known as acute febrile neutrophilic dermatosis, is characterized by sudden-onset fever, neutrophilia, and erythematous painful macules, papules, and nodules on the whole body. This condition is a characteristic neutrophilic dermatoses, and its histopathologic presentation demonstrates dense diffuse infiltrate of neutrophils and massive edema of the papillary dermis. ${ }^{1}$ The pathogenesis is unclear and multifocal but is associated with cytokine dysregulation caused by infection, malignancy, or medication. ${ }^{2}$

Sweet syndrome is also a rare extraintestinal manifestation (EIM) of inflammatory bowel disease (IBD) that can be controlled by anti-tumor necrosis factor (TNF) agents. ${ }^{3-5}$ Interestingly, some studies have reported that Sweet syndrome can develop in patients with IBD being treated with anti-TNF therapy. ${ }^{6,7}$ The objective of this clinical case study is to compare anti-TNF-agent-induced Sweet syndrome with EIMs of IBD. This case may aid medical physicians in determining whether to discontinue anti-TNF agents when facing the dilemma of this differential diagnosis. We also review articles about the anti-TNF-agent-induced paradoxical effect compared with our case.

Case presentation

A 62-year-old male patient with ulcerative colitis presented with diffuse generalized skin rashes and a lowgrade fever after one month of adalimumab therapy. He was concurrently receiving systemic prednisone and mesalazine.

Three months prior, the patient presented to a gastroenterology outpatient department with a one-week history of frequent bloody diarrhea ( $>20$ times per day), severe abdominal cramps, and fever. Examination revealed tenderness and guarding in the left lower abdominal quadrant. Colonoscopy revealed ulceration involving the rectum, entire sigmoid, and left to ascending colon. Multiple biopsies of the colon revealed neutrophilic infiltration in the lamina propria with cryptitis. He responded well to prednisolone and mesalazine for ulcerative colitis. However, prednisolone was not weaned to $10 \mathrm{mg}$ daily, and he was re-admitted to the hospital due to flare-up bowel symptoms. He responded well to high-dose steroids (40 $\mathrm{mg}$ of intravenous methylprednisolone every 12 hours). After discharge, adalimumab (initial dose of $160 \mathrm{mg}$ on September 21, $2020,2^{\text {nd }}$ dose of $80 \mathrm{mg} 2$ weeks later, and $3^{\text {rd }}$ dose of $40 \mathrm{mg}$ one month later) was added to his treatment with daily prednisolone $(20 \mathrm{mg})$ and mesalazine $(2400 \mathrm{mg})$. After one month of these concurrent treatments (with prednisolone tapered from $20 \mathrm{mg}$ to $5 \mathrm{mg}$ to $2.5 \mathrm{mg}$ ), he suffered from diffuse generalized rashes, malaise, and a low-grade fever $\left(37.8^{\circ} \mathrm{C}\right)$.

Physical examination revealed multiple tender erythematous papules, macules, and plaques involving his face, back, upper and lower extremities, palms, and soles (Fig. 1). Laboratory investigations revealed a leukocytosis with 10,200/uL leukocytes, peripheral neutrophilia with $75.6 \%$ neutrophils, and elevated inflammatory markers with positive C-reactive protein. Skin biopsy of his left hand revealed edematous change on the dermal papillae and diffuse inflammatory infiltration in the upper dermis, perivascular and periadnexal areas, and deep dermis, predominantly composed of neutrophils (Fig. 2). A few scattered eosinophils in the interstitial tissue were noted. No definite leukocytoclastic vasculitis was seen. Direct immunofluorescence for IgG, IgA and C3 were negative, and no pathogens were detected by periodic acid-Schiff (PAS) stain.

Based on the diagnostic criteria, a definitive diagnosis of neutrophilic dermatosis (Sweet syndrome) was made. After increasing the steroid dose and discontinuing adalimumab, he had marked symptom improvement in 
five days (Fig. 3). No recurrent symptoms of skin or IBD occurred within 6 months after weaning the prednisolone dose to $2.5 \mathrm{mg}$.

Discussion

Sweet syndrome is known as an EIM and a rare natural course of IBD. ${ }^{8}$ With increasing use of anti-TNF agents to treat IBD, more cutaneous adverse events have been reported, of which the prevalence among patients with IBD ranges between $5 \%$ and $10 \%{ }^{9}$ Some cases of anti-TNF-induced Sweet syndrome have been reported, while some cases of Sweet syndrome as an EIM of IBD treated with anti-TNF agents have been reported. ${ }^{3-7}$ Considering that Sweet syndrome is treated or triggered by anti-TNF agents, medical physicians must decide whether discontinue anti-TNF agents.

The origin of Sweet syndrome in this case was unclear, and the probable causes included EIMs of IBD or medication (adalimumab). Prednisolone could not be tapered to $10 \mathrm{mg}$ daily, and the patient was admitted due to a flare of ulcerative colitis at the first hospitalization. After discharge, he responded well to prednisolone, mesalazine, and adalimumab, which was newly added. Unfortunately, when prednisolone was tapered to 2.5 $\mathrm{mg}$, he presented with multiple polymorphic skin rashes on the whole body. With the previous failure to taper prednisolone, an EIM and flare of ulcerative colitis was suspected initially because cutaneous manifestations parallel IBD activity. ${ }^{8}$ However, drug-induced skin lesions were probable owing to the temporal relationship between the adalimumab treatment and clinical presentation. A marked improvement in skin lesions was observed by increasing the steroid dose and discontinuing adalimumab. Furthermore, he did not suffer from bowel symptoms and skin manifestations for 2 months after weaning prednisolone to 2.5 mg without adalimumab. According to the diagnostic criteria for drug-induced Sweet syndrome, adalimumab-induced Sweet syndrome was suspected because all five of the following criteria were met: abrupt onset of painful skin lesions, histopathologic evidence, pyrexia, temporal relationship between drug ingestion and clinical presentation, and improvement in skin lesions after treatment with corticosteroids and drug withdrawal. ${ }^{10}$

Among reported cases of adalimumab-induced Sweet syndrome, the period from adalimumab administration to skin lesion appearance ranged from 3 to 14 months; thus, differential diagnosis by presentation and resolution alone is difficult. ${ }^{6,7}$ Anti-TNF agents should be discontinued and steroid doses increased in cases of probable anti-TNF-agent-induced Sweet syndrome.

$\mathrm{TNF} \alpha$ is a proinflammatory cytokines, and anti-TNF agents are breakthrough therapeutic treatments for inflammatory disease. However, numerous paradoxical effects including psoriasiform skin lesions have been reported. ${ }^{9}$ he mechanisms of these paradoxical reactions remain controversial. One hypothesis is that inhibiting TNF induces interferon (IFN) production, which might lead to psoriasis. ${ }^{11,12}$ Another is that TNF blockade has an immunoregulatory role in decreasing expansion of Treg cells and increasing Th17 function, leading to exacerbated skin inflammation. ${ }^{2,13}$ These mechanisms are associated with cytokine imbalance under anti-TNF treatment. There are few cases of adalimumab-induced Sweet syndrome. ${ }^{6,7}$ The molecular and cellular mechanisms and pathogenesis of Sweet syndrome are unclear; ${ }^{2}$ however, cytokine dysregulation is suspected. ${ }^{14}$ In summary, Sweet syndrome may be triggered in some patients with cytokine dysregulation caused by anti-TNF agents; in other patients, Sweet syndrome may be an EIM controlled by anti-TNF agents.

A limitation of this study is that the histopathologic evidence was atypical. Skin biopsy revealed characteristic edematous change of the dermal papillae and diffuse inflammatory infiltration in the dermis. However, the neutrophilic infiltration was not as dense as typical patterns. Even though clinicopathological presentations in different categories of Sweet syndrome are similar, there are slight variations owing to the different underlying etiologies including infection, medication, and malignancy. ${ }^{10}$ A significant increase in Th1 cytokines in classic Sweet syndrome leads to neutrophil activation and localization, while a remarkable presence of Th17 cytokines in anti-TNF-agent-induced Sweet syndrome creates an inflammatory response and localizes neutrophils by producing multiple inflammatory molecules and inducing adhesion molecules. ${ }^{2,} 13$ Moreover, neutrophilia is uncommonly present in drug-induced Sweet syndrome. ${ }^{15}$ The intensity of neutrophilic infiltration and adhesion formation may be related to different immunologic pathways leading to the same endpoint 
of leukocytosis. The study of different pathological patterns including classical, malignancy-associated, and drug-induced Sweet syndrome is limited, and further study should be conducted for differential diagnosis.

When distinguishing between EIM flare-ups and drug-induced skin lesions, anti-TNF agents should be withheld, and steroid doses increased in cases of suspected anti-TNF-agent-induced Sweet syndrome. In addition, determining the temporal relationship between drug ingestion and clinical presentation is pivotal. In appropriate situations, patients can be re-challenged to confirm a definitive diagnosis drug-induced Sweet syndrome. Further studies should be conducted to clarify the relationship of cytokine dysregulation between Sweet syndrome and the paradoxical effects of anti-TNF agents to elucidate which patients are vulnerable to Sweet syndrome during anti-TNF therapy.

(e) Acknowledgments

We sincerely thank the patient. We thank all the nursing staff, who provided expertise and care for patients. This study received no specific funding. Both authors are the patients' doctors. All Authors read and approved the manuscript

(f) Conflict of interest statement

The authors declare no conflict of interest.

(g) ORCID number (Yung-Chun Chang): 0000-0001-9658-7923

Corresponding Author (Hui-Ju Yang): 0000-0002-0193-4687

(h) Funding source: no specific funding

(i) Data sharing statement: All data are available to facilitate research reproducibility

(j) Authorship

YCC: primary author of the final manuscript, writing the manuscript and reviewing it; YCC, HJY: involved in study concept; HJY: contributed with pathological expertise and images and revised the manuscript, involved in critical revision

(k) Ethical statement

An informed consent was obtained. All procedures performed in this study were following the ethical standards of the national research committee.

(m) References

1. Cohen PR. Sweet's syndrome-a comprehensive review of an acute febrile neutrophilic dermatosis. Orphanet journal of rare diseases . 2007;2(1):1-28.

2. Heath MS, Ortega-Loayza AG. Insights into the pathogenesis of sweet's syndrome. Frontiers in immunology . 2019;10:414.

3. Yamauchi PS, Turner L, Lowe NJ, Gindi V, Jackson JM. Treatment of recurrent Sweet's syndrome with coexisting rheumatoid arthritis with the tumor necrosis factor antagonist etanercept. Journal of the American Academy of Dermatology . 2006;54(3):S122-S126.

4. Rahier J-F, Lion L, Dewit O, Lambert M. Regression of Sweet's syndrome associated with Crohn's disease after anti-Tumour Necrosis Factor therapy. Acta gastro-enterologica Belgica . 2005;68(3):376-379.

5. Foster EN, Nguyen KK, Sheikh RA, Prindiville TP. Crohn's disease associated with Sweet's syndrome and Sjögren's syndrome treated with infliximab. Clinical and Developmental Immunology . 2005;12(2):145-149.

6. Hawryluk EB, Linskey KR, Duncan LM, Nazarian RM. Broad range of adverse cutaneous eruptions in patients on TNF-alpha antagonists. Journal of cutaneous pathology . 2012;39(5):481-492. 
7. Banse C, Sobocinski V, Savoye G, Avenel G, Vittecoq O. Occurrence of Sweet syndrome under anti-TNF. Clinical rheumatology . 2015;34(11):1993-1994.

8. Greuter T, Navarini A, Vavricka SR. Skin manifestations of inflammatory bowel disease. Clinical reviews in allergy $\mathcal{E} 3$ immunology . 2017;53(3):413-427.

9. Fiorino G, Danese S, Pariente B, Allez M. Paradoxical immune-mediated inflammation in inflammatory bowel disease patients receiving anti-TNF- $\alpha$ agents. Autoimmunity reviews . 2014;13(1):15-19.

10. von den Driesch P. Sweet's syndrome (acute febrile neutrophilic dermatosis). Journal of the American Academy of Dermatology . 1994;31(4):535-556.

11. de Gannes GC, Ghoreishi M, Pope J, et al. Psoriasis and pustular dermatitis triggered by TNF- $\alpha$ inhibitors in patients with rheumatologic conditions. Archives of Dermatology . 2007;143(2):223-231.

12. Seneschal J, Milpied B, Vergier B, Lepreux S, Schaeverbeke T, Taïeb A. Cytokine imbalance with increased production of interferon- $\alpha$ in psoriasiform eruptions associated with antitumour necrosis factor- $\alpha$ treatments. British Journal of Dermatology . 2009;161(5):1081-1088.

13. Ma HL, Napierata L, Stedman N, et al. Tumor necrosis factor $\alpha$ blockade exacerbates murine psoriasislike disease by enhancing Th17 function and decreasing expansion of Treg cells. Arthritis 83 Rheumatism: Official Journal of the American College of Rheumatology . 2010;62(2):430-440.

14. Giasuddin AS, El-Orfi AHA, Ziu MM, El-Barnawi NY. Sweet's syndrome: is the pathogenesis mediated by helper T cell type 1 cytokines? Journal of the American Academy of Dermatology . 1998;39(6):940-943.

15. Walker DC, Cohen PR. Trimethoprim-sulfamethoxazole-associated acute febrile neutrophilic dermatosis: case report and review of drug-induced Sweet's syndrome. Journal of the American Academy of Dermatology . 1996;34(5):918-923.

(l) Figure legends

1. Figure 1: Initial clinical presentation. Multiple painful erythematous papules, macules, and plaques involving the (a) back, (b) palm, and (c) lower extremities after adalimumab therapy.

2. Figure 2: Histopathological presentation. (a) A low magnification shows diffuse inflammatory infiltrate in the upper dermis and deep dermis. (hematoxylin and eosin stain $[\mathrm{H} \& \mathrm{E}], \mathrm{x} 40$ magnification) (b) An intermediate magnification shows prominent papillary dermal edematous change. (H\&E, x100 magnification) (c) A high magnification view shows perivascular and periadnexal lymphocytic infiltrate, predominantly composed of neutrophils. A few scattered eosinophils are noted and no definite leukocytoclastic vasculitis is found. (H\&E, x400 magnification)

3. Figure 3: (a-c) Marked lesion improvement after two days of withholding adalimumab and increasing the steroid dose.
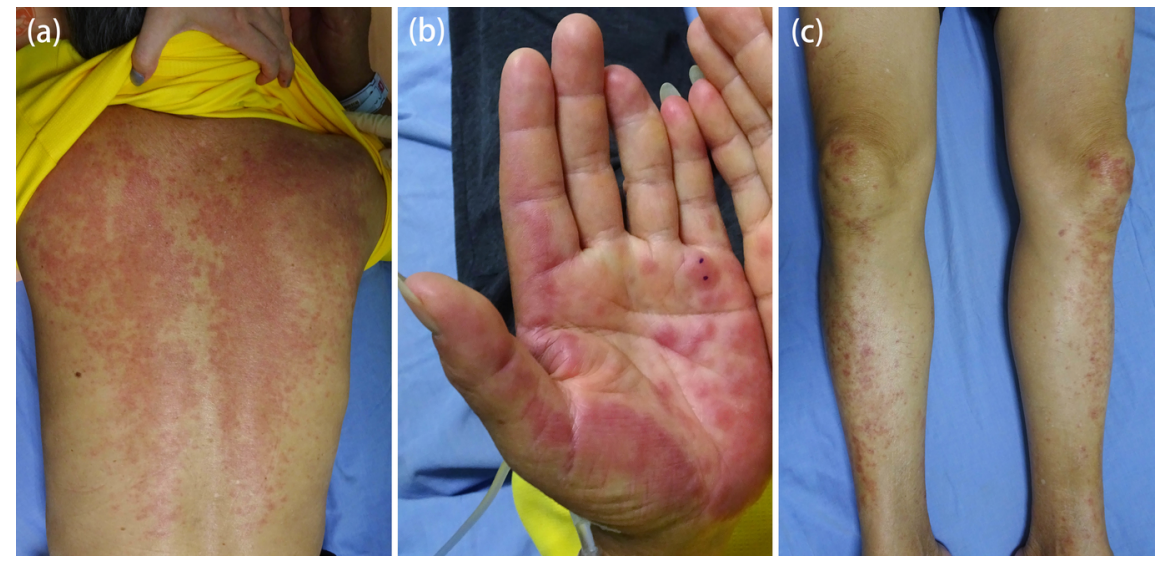

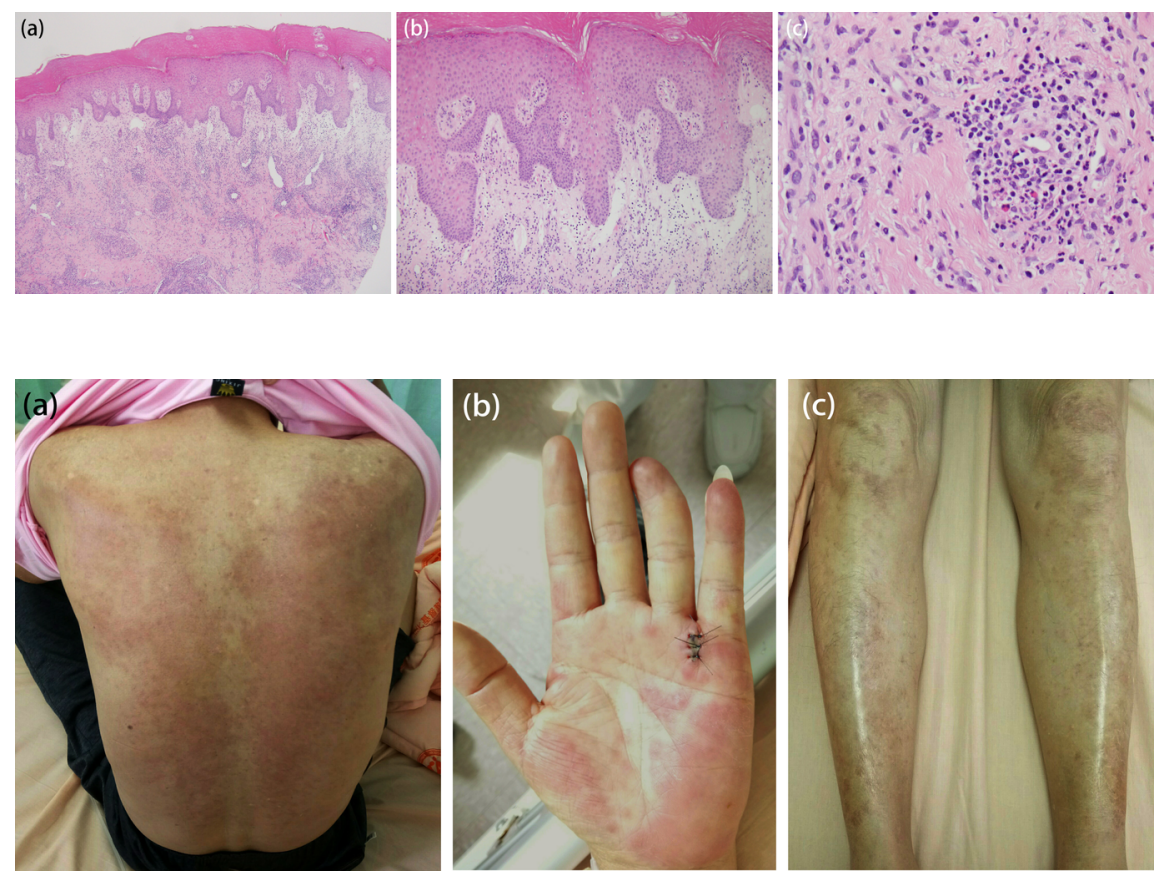